Proyecciones Journal of Mathematics

Vol. 31, No 4, pp. 373-390, December 2012.

Universidad Católica del Norte

Antofagasta - Chile

\title{
Lacunary generalized difference statistical convergence in random 2-normed spaces
}

\author{
Bipan Hazarika \\ Rajiv Gandhi University, India \\ Received : August 2012. Accepted : September 2012
}

\begin{abstract}
Recently in [22], Mursaleen introduced the concept of statistical convergence in random 2-normed spaces. In this paper, we define and study the notion of lacunary $\Delta^{n}$-statistical convergence and lacunary $\Delta^{n}$-statistical Cauchy sequences in random 2-normed spaces using lacunary density and prove some interesting theorems.
\end{abstract}

Subjclass [2000] : Primary 40A05; Secondary 46A70, 40A99, 46499 .

Keywords : Statistical convergence; lacunary sequence; difference sequence; t-norm; 2-norm; random 2-normed space. 


\section{Introduction}

The concept of statistical convergence play a vital role not only in pure mathematics but also in other branches of science involving mathematics, especially in information theory, computer science, biological science, dynamical systems, geographic information systems, population modelling, and motion planning in robotics.

The notion of statistical convergence was introduced by Fast [7] and Schoenberg [27] independently. A lot of developments have been made in this areas after the works of Salát [26], Fridy [8] and Miller [21]. Over the years and under different names statistical convergence has been discussed in the theory of Fourier analysis, ergodic theory and number theory. Fridy and Orhan [9] introduced the concept of lacunary statistical convergence. In [23], Mursaleen and Mohiuddine introduced the concept of lacunary statistical convergence with respect to the intuitionistic fuzzy normed space. Some work on lacunary statistical convergence can be found in [2], [10], $[19],[25]$. In the recent years, generalization of statistical convergence have appeared in the study of strong integral summability and the structure of ideals of bounded continuous functions on Stone-Čech compactification of the natural numbers. Moreover statistical convergence is closely related to the concept of convergence in probability, (see [3]).

The probabilistic metric space was introduced by Menger [20] which is an interesting and important generalization of the notion of a metric space. Karakus [17] studied the concept of statistical convergence in probabilistic normed spaces. The theory of probabilistic normed(or metric) spaces was initiated and developed in [1], [28], [29], [30], [31] and further it was extended to random/probabilistic 2-normed spaces by Golet [13] using the concept of 2-norm which is defined by Gähler [11], and Gürdal and Pehlivan [15] studied statistical convergence in 2-Banach spaces.

The notion of statistical convergence depends on the density of subsets of $\mathbf{N}$. A subset of $\mathbf{N}$ is said to have density $\delta(E)$ if

$$
\delta(E)=\lim _{n \rightarrow \infty} \frac{1}{n}|\{k \leq n: k \in E\}|,
$$

where the vertical bars denote the cardinality of the enclosed set. 
A single sequence $x=\left(x_{k}\right)$ is said to be statistically convergent to $\ell$ if for every $\varepsilon>0$

$$
\delta\left(\left\{k \in \mathbf{N}:\left|x_{k}-\ell\right| \geq \varepsilon\right\}\right)=0 .
$$

In this case we write $S$-lim $x=\ell$ or $x_{k} \rightarrow \ell(S)$ (see [7],[8]).

A sequence $x=\left(x_{k}\right)$ is said to be $\Delta^{n}$-satistically convergent to $\ell$ if for every $\varepsilon>0$ the set $\left\{k \in \mathbf{N}:\left|\Delta^{n} x_{k}-\ell\right| \geq \varepsilon\right\}$ has natural density zero (see $[6])$. i.e.

$$
\lim _{m \rightarrow \infty} \frac{1}{m}\left|\left\{k \leq m:\left|\Delta^{n} x_{k}-\ell\right| \geq \varepsilon\right\}\right|=0,
$$

where $n \in \mathbf{N}$ and $\Delta^{0} x_{k}=\left(x_{k}\right), \Delta x_{k}=\left(x_{k}-x_{k+1}\right), \Delta^{n} x_{k}=\left(\Delta^{n} x_{k}\right)=$ $\left(\Delta^{n-1} x_{k}-\Delta^{n-1} x_{k+1}\right)$, and also this generalized difference notion has the following binomial representation:

$$
\Delta^{n} x_{k}=\sum_{i=0}^{n}(-1)^{i}\left(\begin{array}{c}
n \\
i
\end{array}\right) x_{k+i} \text { for all } k \in \mathbf{N} .
$$

\section{Preliminaries}

Definition 2.1. A function $f: \mathbf{R} \rightarrow \mathbf{R}_{0}^{+}$is called a distribution function if it is a non-decreasing and left continuous with $\inf _{t \in \mathbf{R}} f(t)=0$ and $\sup _{t \in \mathbf{R}} f(t)=1$. By $D^{+}$, we denote the set of all distribution functions such that $f(0)=0$. If $a \in \mathbf{R}_{0}^{+}$, then $H_{a} \in D^{+}$, where

$$
H_{a}(t)= \begin{cases}1, & \text { if } t>a \\ 0, & \text { if } t \leq a\end{cases}
$$

It is obvious that $H_{0} \geq f$ for all $f \in D^{+}$.

A $t$-norm is a continuous mapping $*:[0,1] \times[0,1] \rightarrow[0,1]$ such that $([0,1], *)$ is abelian monoid with unit one and $c * d \geq a * b$ if $c \geq a$ and $d \geq b$ for all $a, b, c \in[0,1]$. A triangle function $\tau$ is a binary operation on $D^{+}$, which is commutative, associative and $\tau\left(f, H_{0}\right)=f$ for every $f \in D^{+}$.

In [11],Gähler introduced the following concept of 2-normed spaces.

Definition 2.2. Let $X$ be a linear space of dimension $d>1$ ( $d$ may be infinite). A real-valued function $\|.,$.$\| from X^{2}$ into $\mathbf{R}$ satisfying the following conditions: 
(1) $\left\|x_{1}, x_{2}\right\|=0$ if and only if $x_{1}, x_{2}$ are linearly dependent,

(2) $\left\|x_{1}, x_{2}\right\|$ is invariant under permutation,

(3) $\left\|\alpha x_{1}, x_{2}\right\|=|\alpha|\left\|x_{1}, x_{2}\right\|$, for any $\alpha \in \mathbf{R}$,

(4) $\left\|x+\bar{x}, x_{2}\right\| \leq\left\|x, x_{2}\right\|+\left\|\bar{x}, x_{2}\right\|$

is called an 2-norm on $X$ and the pair $(X,\|.,\|$.$) is called an 2-normed space.$

A trivial example of an 2-normed space is $X=\mathbf{R}^{2}$, equipped with the Euclidean 2-norm $\left\|x_{1}, x_{2}\right\|_{E}=$ the volume of the parallellogram spanned by the vectors $x_{1}, x_{2}$ which may be given expicitly by the formula

$$
\left\|x_{1}, x_{2}\right\|_{E}=\left|\operatorname{det}\left(x_{i j}\right)\right|=a b s\left(\operatorname{det}\left(<x_{i}, x_{j}>\right)\right)
$$

where $x_{i}=\left(x_{i 1}, x_{i 2}\right) \in \mathbf{R}^{2}$ for each $i=1,2$.

Recently, Goleț [13] used the idea of 2-normed space to define the random 2-normed spaces.

Definition 2.3. Let $X$ be a linear space of dimension $d>1$ ( $d$ may be infinite), $\tau$ a triangle, and $\mathcal{F}: X \times X \rightarrow D^{+}$. Then $\mathcal{F}$ is called a probabilistic 2-norm and $(X, \mathcal{F}, \tau)$ a probabilistic 2-normed space if the following conditions are satisfied:

$\left(P 2 N_{1}\right) \mathcal{F}(x, y ; t)=H_{0}(t)$ if $x$ and $y$ are linearly dependent, where $\mathcal{F}(x, y ; t)$ denotes the value of $\mathcal{F}(x, y)$ at $t \in \mathbf{R}$,

$\left(P 2 N_{2}\right) \mathcal{F}(x, y ; t) \neq H_{0}(t)$ if $x$ and $y$ are linearly independent,

$\left(P 2 N_{3}\right) \mathcal{F}(x, y ; t)=\mathcal{F}(y, x ; t)$, for all $x, y \in X$,

$\left(P 2 N_{4}\right) \mathcal{F}(\alpha x, y ; t)=\mathcal{F}\left(x, y ; \frac{t}{|\alpha|}\right)$, for every $t>0, \alpha \neq 0$ and $x, y \in X$,

$\left(P 2 N_{5}\right) \mathcal{F}(x+y, z ; t) \geq \tau(\mathcal{F}(x, z ; t), \mathcal{F}(y, z ; t))$, whenever $x, y, z \in X$.

If $\left(P 2 N_{5}\right)$ is replaced by

$\left(P 2 N_{6}\right) \mathcal{F}\left(x+y, z ; t_{1}+t_{2}\right) \geq \mathcal{F}\left(x, z ; t_{1}\right) * \mathcal{F}\left(y, z ; t_{2}\right)$, for all $x, y, z \in X$ and $t_{1}, t_{2} \in \mathbf{R}_{0}^{+}$

then $(X, \mathcal{F}, *)$ is called a random 2-normed space (for short, R2NS).

Remark 2.1. $\quad$ Every 2-normed space $(X,\|.,\|$.$) can be made a random$ 2-normed space in a natural way, by setting

$(i) \mathcal{F}(x, y ; t)=H_{0}(t-\|x, y\|)$, for every $x, y \in X, t>0$ and $a * b=$ $\min \{a, b\}, a, b \in[0,1]$;

(ii) $\mathcal{F}(x, y ; t)=\frac{t}{t+\|x, y\|}$, for every $x, y \in X, t>0$ and $a * b=a b, a, b \in[0,1]$.

Definition 2.4. A sequence $x=\left(x_{k}\right)$ in a random 2-normed space $(X, \mathcal{F}, *)$ is said to be convergent (or $\mathcal{F}$-convergent) to $\ell \in X$ with respect to $\mathcal{F}$ if 
for each $\varepsilon>0, \eta \in(0,1)$ and non zero $z \in X$ there exists an positive integer $n_{0}=n_{0}(\varepsilon, z)$ such that $\mathcal{F}\left(x_{k}-\ell, z ; \varepsilon\right)>1-\eta$, whenever $k \geq n_{0}$. In this case we write $\mathcal{F}-\lim _{k} x_{k}=\ell$, and $\ell$ is called the $\mathcal{F}$-limit of $x=\left(x_{k}\right)$.

Definition 2.5. A sequence $x=\left(x_{k}\right)$ in a random 2-normed space $(X, \mathcal{F}, *)$ is said to be Cauchy with respect to $\mathcal{F}$ if for each $\varepsilon>0, \eta \in(0,1)$ and non zero $z \in X$, there exists a positive integer $n_{0}=n_{0}(\varepsilon, z)$ such that $\mathcal{F}\left(x_{k}-x_{m}, z ; \varepsilon\right)>1-\eta$, whenever $k, m \geq n_{0}$.

In [14], Gürdal and Pehlivan studied statistical convergence in 2-normed spaces and in 2-Banach spaces in [15]. In fact, Mursaleen [22] studied the concept of statistical convergence of sequences in random 2-normed spaces, in [24], Mohiuddine and Aiyub introduced the concept of lacunary statistical convergence in random 2-normed space. Recently in [4], Esi and Özdemir introduced and studied the concept of generalized $\Delta^{m}$-statistical convergence of sequences in probabilistic normed spaces and in [5] Esi and Özdemir introduced and studied the concept of lacunary statistical convergence in random n-normed space.

Definition 2.6. [22] A sequence $x=\left(x_{k}\right)$ in a random 2-normed space $(X, \mathcal{F}, *)$ is said to be statistical-convergent or $S^{R 2 N}$-convergent to some $\ell \in X$ with respect to $\mathcal{F}$ if for each $\varepsilon>0, \eta \in(0,1)$ and non zero $z \in X$ such that

$$
\delta\left(\left\{k \in \mathbf{N}: \mathcal{F}\left(x_{k}-\ell, z ; \varepsilon\right) \leq 1-\eta\right\}\right)=0,
$$

In other words we can write the sequence $\left(x_{k}\right)$ statistical converges to $\ell$ in random 2-normed space $(X, \mathcal{F}, *)$ if

$$
\lim _{m \rightarrow \infty} \frac{1}{m}\left|\left\{k \leq m: \mathcal{F}\left(x_{k}-\ell, z ; \varepsilon\right) \leq 1-\eta\right\}\right|=0 .
$$

or equivalently

$$
\delta\left(\left\{k \in \mathbf{N}: \mathcal{F}\left(x_{k}-\ell, z ; \varepsilon\right)>1-\eta\right\}\right)=1,
$$

i.e.

$$
S-\lim _{k \rightarrow \infty} \mathcal{F}\left(x_{k}-\ell, z ; \varepsilon\right)=1 .
$$

In this case we write $S^{R 2 N}-\lim x=\ell$ and $\ell$ is called the $S^{R 2 N}-$ limit of $x$. Let $S^{R 2 N}(X)$ denotes the set of all statistical convergent sequences in random 2-normed space $(X, \mathcal{F}, *)$. 
In this paper we define and study lacunary $\Delta^{n}$-statistical convergence in random 2-normed space which is quite a new and interesting idea to work with. We show that some properties of lacunary $\Delta^{n}$-statistical convergence of real numbers also hold for sequences in random 2-normed spaces. We find some relations related to lacunary $\Delta^{n}$-statistical convergent sequences in random 2-normed spaces. Also we find out the relation between lacunary $\Delta^{n}$-statistical convergent and lacunary $\Delta^{n}$-statistical Cauchy sequences in this spaces.

\section{Lacunary $\Delta^{n}$-statistical convergence in random 2-normed spaces}

In this section we define lacunary $\Delta^{n}$-statistical convergent sequence in random 2-normed $(X, \mathcal{F}, *)$. Also we obtained some basic properties of this notion in random 2-normed spaces.

Definition 3.1. By a lacunary sequence $\theta=\left(k_{r}\right)$, where $k_{0}=0$, we shall mean an increasing sequence of non-negative integers with $h_{r}: k_{r}-$ $k_{r-1} \rightarrow \infty$ as $r \rightarrow \infty$. The intervals determined by $\theta$ will be denoted by $I_{r}=\left(k_{r-1}, k_{r}\right]$ and the ratio $\frac{k_{r}}{k_{r-1}}$ will be defined by $q_{r}$.

Let $\theta$ be a lacunary sequence and $I_{r}=\left\{k: k_{r-1}<k \leq k_{r}\right\}$. A set $K \subset \mathbf{N}$ has lacunary density $\delta_{\theta}(K)$ if

$$
\lim _{r} \frac{1}{h_{r}}\left|\left\{i \in I_{r}: i \in K\right\}\right|=0 .
$$

Definition 3.2. Let $\theta$ be a lacunary sequence. A sequence $x=\left(x_{k}\right)$ is said to be $S_{\theta}$-convergent to $\ell$ provided that for each $\varepsilon>0$, the set

$$
K(\varepsilon)=\left\{k \in \mathbf{N}:\left|x_{k}-\ell\right| \geq \varepsilon\right\}
$$

has $\theta$-density zero. In this case we write $S_{\theta}-\lim x=\ell$ or $x_{k} \rightarrow \ell\left(S_{\theta}\right)$ (for details see [9], [10]).

To examine the above notion we will provide some examples.

Example 3.1. Let $\theta=\left(2^{r}-1\right)$ and $K=\left\{i^{2}: i \in \mathbf{N}\right\}$. Then we have

$$
\delta_{\theta}(K)=0=\delta(K) \text {. }
$$

Example 3.2. Let $\theta=\left(2^{r}-1\right)$ and $K=\{i: i \in \mathbf{N}\}$. Then we have $\delta_{\theta}(K)=0$. But $\delta(K)=1$. 
We define the $\Delta^{n}$-convergence in random 2-normed spaces as follows:

Definition 3.3. A sequence $x=\left(x_{k}\right)$ in a random 2-normed space $(X, \mathcal{F}, *)$ is said to be $\Delta^{n}$-convergent to $\ell \in X$ with respect to $\mathcal{F}$ if for each $\varepsilon>0$, $\eta \in(0,1)$ and non zero $z \in X$, there exists an positive integer $n_{0}=n_{0}(\varepsilon, z)$ such that $\mathcal{F}\left(\Delta^{n} x_{k}-\ell, z ; \varepsilon\right)>1-\eta$, whenever $k \geq n_{0}$. In this case we write $\mathcal{F}-\lim _{k} \Delta^{n} x_{k}=\ell$, and $\ell$ is called the $\mathcal{F}_{\Delta^{n}}$-limit of $x=\left(x_{k}\right)$.

Definition 3.4. A sequence $x=\left(x_{k}\right)$ in a random 2-normed space $(X, \mathcal{F}, *)$ is said to be $\Delta^{n}$-Cauchy with respect to $\mathcal{F}$ if for each $\varepsilon>0, \eta \in(0,1)$ and non zero $z \in X$, there exists a positive integer $n_{0}=n_{0}(\varepsilon, z)$ such that $\mathcal{F}\left(\Delta^{n} x_{k}-\Delta^{n} x_{s}, z ; \varepsilon\right)>1-\eta$, whenever $k, s \geq n_{0}$.

In [16] Hazarika and Savas and [24] Mohiuddine and Aiyub independently introduced lacunary satistically convergence in random 2-normed spaces as follows.

Definition 3.5. ([16], [24]) A sequence $x=\left(x_{k}\right)$ in a random 2-normed space $(X, \mathcal{F}, *)$ is said to be lacunary satistically convergent or $S_{\theta^{-}}$convergent to $\ell \in X$ with respect to $\mathcal{F}$ if for every $\varepsilon>0, \eta \in(0,1)$ and non zero $z \in X$ such that

$$
\delta_{\theta}\left(\left\{k \in \mathbf{N}: \mathcal{F}\left(x_{k}-\ell, z ; \varepsilon\right) \leq 1-\eta\right\}\right)=0 .
$$

or equivalently

$$
\delta_{\theta}\left(\left\{k \in \mathbf{N}: \mathcal{F}\left(x_{k}-\ell, z ; \varepsilon\right)>1-\eta\right\}\right)=1,
$$

i.e.

$$
S_{\theta}-\lim _{k \rightarrow \infty} \mathcal{F}\left(x_{k}-\ell, z ; \varepsilon\right)=1 .
$$

In this case we write $S_{\theta}^{R 2 N}-\lim x=\ell$ or $x_{k} \rightarrow \ell\left(S_{\theta}^{R 2 N}\right)$ and

$$
S_{\theta}^{R 2 N}(X)=\left\{x=\left(x_{k}\right): \exists \ell \in \mathbf{R}, S_{\theta}^{R 2 N}-\lim x=\ell\right\} .
$$

Let $S_{\theta}^{R 2 N}(X)$ denotes the set of all lacunary statistical convergent sequences in random 2-normed space $(X, \mathcal{F}, *)$.

Definition 3.6. ([16], [24]) A sequence $x=\left(x_{k}\right)$ in a random 2-normed space $(X, \mathcal{F}, *)$ is said to be lacunary statistical Cauchy with respect to $\mathcal{F}$ if for each $\varepsilon>0, \eta \in(0,1)$ and non zero $z \in X$ there exists a positive integer $n=n(\varepsilon, z)$ such that

$$
\delta_{\theta}\left(\left\{k \in \mathbf{N}: \mathcal{F}\left(x_{k}-x_{n}, z ; \varepsilon\right) \leq 1-\eta\right\}\right)=0 .
$$


or equivalently

$$
\delta_{\theta}\left(\left\{k \in \mathbf{N}: \mathcal{F}\left(x_{k}-x_{n}, z ; \varepsilon\right)>1-\eta\right\}\right)=1 .
$$

Now, we define the lacunary $\Delta^{n}$-statistically convergence in random 2-normed spaces.

Definition 3.7. A sequence $x=\left(x_{k}\right)$ in a random 2-normed space $(X, \mathcal{F}, *)$ is said to be lacunary $\Delta^{n}$-satistically convergent or $S_{\theta\left(\Delta^{n}\right) \text {-convergent to }}$ $\ell \in X$ with respect to $\mathcal{F}$ if for every $\varepsilon>0, \eta \in(0,1)$ and non zero $z \in X$ such that

$$
\delta_{\theta\left(\Delta^{n}\right)}\left(\left\{k \in \mathbf{N}: \mathcal{F}\left(\Delta^{n} x_{k}-\ell, z ; \varepsilon\right) \leq 1-\eta\right\}\right)=0 .
$$

or equivalently

$$
\delta_{\theta\left(\Delta^{n}\right)}\left(\left\{k \in \mathbf{N}: \mathcal{F}\left(\Delta^{n} x_{k}-\ell, z ; \varepsilon\right)>1-\eta\right\}\right)=1,
$$

i.e.

$$
S_{\theta\left(\Delta^{n}\right)}-\lim _{k \rightarrow \infty} \mathcal{F}\left(\Delta^{n} x_{k}-\ell, z ; \varepsilon\right)=1 .
$$

In this case we write $S_{\theta\left(\Delta^{n}\right)}^{R 2 N}-\lim x=\ell$ or $x_{k} \rightarrow \ell\left(S_{\theta\left(\Delta^{n}\right)}^{R 2 N}\right)$ and

$$
S_{\theta\left(\Delta^{n}\right)}^{R 2 N}=\left\{x=\left(x_{k}\right): \exists \ell \in \mathbf{R}, S_{\theta\left(\Delta^{n}\right)}^{R 2 N}-\lim x=\ell\right\} .
$$

Let $S_{\theta\left(\Delta^{n}\right)}^{R 2 N}$ denotes the set of all lacunary $\Delta^{n}$-statistical convergent sequences in random 2-normed space $(X, \mathcal{F}, *)$.

Definition 3.8. A sequence $x=\left(x_{k}\right)$ in a random 2-normed space $(X, \mathcal{F}, *)$ is said to be lacunary $\Delta^{n}$-statistical Cauchy with respect to $\mathcal{F}$ if for every $\varepsilon>0, \eta \in(0,1)$ and non zero $z \in X$ there exists a positive integer $m=$ $m(\varepsilon, z)$ such that for all $k, s \geq m$

$$
\delta_{\theta\left(\Delta^{n}\right)}\left(\left\{k \in \mathbf{N}: \mathcal{F}\left(\Delta^{n} x_{k}-\Delta^{n} x_{s}, z ; \varepsilon\right) \leq 1-\eta\right\}\right)=0 .
$$

or equivalently

$$
\delta_{\theta\left(\Delta^{n}\right)}\left(\left\{k \in \mathbf{N}: \mathcal{F}\left(\Delta^{n} x_{k}-\Delta^{n} x_{s}, z ; \varepsilon\right)>1-\eta\right\}\right)=1 .
$$


Definition 3.7, immediately implies the following Lemma.

Lemma 3.1. Let $(X, \mathcal{F}, *)$ be a random 2-normed space. If $x=\left(x_{k}\right)$ is a sequence in $X$, then for every $\varepsilon>0, \eta \in(0,1)$ and for non zero $z \in X$, then the following statements are equivalent.

(i) $S_{\theta\left(\Delta^{n}\right)}^{R 2 N}-\lim _{k \rightarrow \infty} x_{k}=\ell$.

(ii) $\delta_{\theta\left(\Delta^{n}\right)}\left(\left\{k \in \mathbf{N}: \mathcal{F}\left(\Delta^{n} x_{k}-\ell, z ; \varepsilon\right) \leq 1-\theta\right\}\right)=0$.

(iii) $\delta_{\theta\left(\Delta^{n}\right)}\left(\left\{k \in \mathbf{N}: \mathcal{F}\left(\Delta^{n} x_{k}-\ell, z ; \varepsilon\right)>1-\theta\right\}\right)=1$.

(iv) $S_{\theta\left(\Delta^{n}\right)}-\lim _{k \rightarrow \infty} \mathcal{F}\left(\Delta^{n} x_{k}-\ell, z ; \varepsilon\right)=1$.

Theorem 3.2. Let $(X, \mathcal{F}, *)$ be a random 2-normed space. If $x=\left(x_{k}\right)$ is a sequence in $X$ such that $S_{\theta\left(\Delta^{n}\right)}^{R 2 N}-\lim x_{k}=\ell$ exists, then it is unique.

Proof. Suppose that there exist elements $\ell_{1}, \ell_{2}\left(\ell_{1} \neq \ell_{2}\right)$ in $X$ such that

$$
S_{\theta\left(\Delta^{n}\right)}^{R 2 N}-\lim _{k \rightarrow \infty} x_{k}=\ell_{1} ; S_{\theta\left(\Delta^{n}\right)}^{R 2 N}-\lim _{k \rightarrow \infty} x_{k}=\ell_{2} .
$$

Let $\varepsilon>0$ be given. Choose $a>0$ such that

$$
(1-a) *(1-a)>1-\varepsilon .
$$

Then, for any $t>0$ and for non zero $z \in X$ we define

$$
\begin{aligned}
& K_{1}(a, t)=\left\{k \in \mathbf{N}: \mathcal{F}\left(\Delta^{n} x_{k}-\ell_{1}, z ; \frac{t}{2}\right) \leq 1-a\right\} ; \\
& K_{2}(a, t)=\left\{k \in \mathbf{N}: \mathcal{F}\left(\Delta^{n} x_{k}-\ell_{2}, z ; \frac{t}{2}\right) \leq 1-a\right\} .
\end{aligned}
$$

Since

$S_{\theta\left(\Delta^{n}\right)}^{R 2 N}-\lim _{k \rightarrow \infty} x_{k}=\ell_{1}$ and $S_{\theta\left(\Delta^{n}\right)}^{R 2 N}-\lim _{k \rightarrow \infty} x_{k}=\ell_{2}$, we have

$\delta_{\theta\left(\Delta^{n}\right)}\left(K_{1}(a, t)\right)=0$ and $\delta_{\theta\left(\Delta^{n}\right)}\left(K_{2}(a, t)\right)=0$ for all $t>0$.

Now let $K(a, t)=K_{1}(a, t) \cup K_{2}(a, t)$, then it is easy to observe that $\delta_{\theta\left(\Delta^{n}\right)}(K(a, t))=0$. But we have $\delta_{\theta\left(\Delta^{n}\right)}\left(K^{c}(a, t)\right)=1$.

Now if $k \in K^{c}(a, t)$ then we have

$$
\mathcal{F}\left(\ell_{1}-\ell_{2}, z ; t\right) \geq \mathcal{F}\left(\Delta^{n} x_{k}-\ell_{1}, z ; \frac{t}{2}\right) * \mathcal{F}\left(\Delta^{n} x_{k}-\ell_{2}, z ; \frac{t}{2}\right)>(1-a) *(1-a) .
$$


It follows by (3.1) that

$$
\mathcal{F}\left(\ell_{1}-\ell_{2}, z ; t\right)>(1-\varepsilon) .
$$

Since $\varepsilon>0$ was arbitrary, we get $\mathcal{F}\left(\ell_{1}-\ell_{2}, z ; t\right)=0$ for all $t>0$ and non zero $z \in X$. Hence $\ell_{1}=\ell_{2}$.

Next theorem gives the algebraic characterization of lacunary $\Delta^{n}$-statistical convergence on random 2-normed spaces.

Theorem 3.3. Let $(X, \mathcal{F}, *)$ be a random 2-normed space, and $x=\left(x_{k}\right)$ and $y=\left(y_{k}\right)$ be two sequences in $X$.

(a) If $S_{\theta\left(\Delta^{n}\right)}^{R 2 N}-\lim x_{k}=\ell$ and $c(\neq 0) \in \mathbf{R}$, then $S_{\theta\left(\Delta^{n}\right)}^{R 2 N}-\lim c x_{k}=c \ell$.

(b) If $S_{\theta\left(\Delta^{n}\right)}^{R 2 N}-\lim x_{k}=\ell_{1}$ and $S_{\theta\left(\Delta^{n}\right)}^{R 2 N}-\lim y_{k}=\ell_{2}$, then $S_{\lambda\left(\Delta^{n}\right)}^{R 2 N}-$ $\lim \left(x_{k}+y_{k}\right)=\ell_{1}+\ell_{2}$.

Proof of the theorem is straightforward, thus omitted.

Theorem 3.4. Let $(X, \mathcal{F}, *)$ be a random 2-normed space. If $x=\left(x_{k}\right)$ be a sequence in $X$ such that $\mathcal{F}_{\Delta^{n}}-\lim x_{k}=\ell$ then $S_{\theta\left(\Delta^{n}\right)}^{R 2 N}-\lim x_{k}=\ell$.

Proof. Let $\mathcal{F}_{\Delta^{n}}-\lim x_{k}=\ell$. Then for every $\varepsilon>0, t>0$ and non zero $z \in X$, there is a positive integer $n_{0}=n_{0}(\varepsilon, z)$ such that

$$
\mathcal{F}\left(\Delta^{n} x_{k}-\ell, z ; t\right)>1-\varepsilon
$$

for all $k \geq n_{0}$. We put

$$
K(\varepsilon, t)=\left\{k \in \mathbf{N}: \mathcal{F}\left(\Delta^{n} x_{k}-\ell, z ; t\right) \leq 1-\varepsilon\right\} .
$$

Also, since every finite subset of $\mathbf{N}$ has $\delta_{\theta\left(\Delta^{n}\right)}$-density zero, and consequently we have $\delta_{\theta\left(\Delta^{n}\right)}(K(\varepsilon, t))=0$. This shows that $S_{\theta\left(\Delta^{n}\right)}^{R 2 N}-\lim x_{k}=\ell$.

Remark 3.5. The converse of the above theorem is not true in general. It follows from the following example.

Example 3.6. Let $X=\mathbf{R}^{2}$, with the 2-norm $\|x, z\|=\left|x_{1} z_{2}-x_{2} z_{1}\right|$, $x=\left(x_{1}, x_{2}\right), z=\left(z_{1}, z_{2}\right)$ and $a * b=a b$ for all $a, b \in[0,1]$. Let $\mathcal{F}(x, y ; t)=$ $\frac{t}{t+\|x, y\|}$, for all $x, z \in X, z_{2} \neq 0$, and $t>0$. Now we define a sequence 
$x=\left(x_{k}\right)$ by

$$
\Delta^{n} x_{k}=\left\{\begin{array}{cc}
(k, 0), & \text { if } k_{r}-\left[\sqrt{h_{r}}\right]+1 \leq k \leq k_{r}, \quad r \in \mathbf{N} ; \\
(0,0), & \text { otherwise. }
\end{array}\right.
$$

Nor for every $0<\varepsilon<1$ and $t>0$, write

$$
\begin{gathered}
K(\varepsilon, t)=\left\{k \in \mathbf{N}: \mathcal{F}\left(\Delta^{n} x_{k}-\ell, z ; t\right) \leq 1-\varepsilon\right\}, \ell=(0,0) \\
=\left\{k \in \mathbf{N}: \frac{t}{t+\left\|x_{k}, z\right\|} \leq 1-\varepsilon\right\}=\left\{k \in \mathbf{N}:\left\|x_{k}, z\right\| \geq \frac{t \varepsilon}{1-\varepsilon}>0\right\} \\
=\left\{k \in \mathbf{N}: k_{r}-\left[\sqrt{h_{r}}\right]+1 \leq k \leq k_{r}, \quad r \in \mathbf{N}\right\},
\end{gathered}
$$

We see that

$$
\frac{1}{h_{r}}|K(\varepsilon, t)| \leq \frac{1}{h_{r}}\left|\left\{k \in \mathbf{N}: k_{r}-\left[\sqrt{h_{r}}\right]+1 \leq k \leq k_{r}, \quad r \in \mathbf{N}\right\}\right| \leq \frac{\left[\sqrt{h_{r}}\right]}{h_{r}} .
$$

Therefore we get

$$
\delta_{\theta\left(\Delta^{n}\right)}(K(\varepsilon, t))=\lim _{r \rightarrow \infty} \frac{\left[\sqrt{h_{r}}\right]}{h_{r}}=0 .
$$

This shows that $S_{\theta\left(\Delta^{n}\right)}^{R 2 N}-\lim x_{k}=0$.

On the other hand the sequence is not $\mathcal{F}$-convergent to zero as

$$
\mathcal{F}\left(x_{k}-\ell, z ; t\right)=\frac{t}{t+\left\|x_{k}, z\right\|}=\left\{\begin{array}{cc}
\frac{t}{t+\left\|x_{k}, z\right\|}, & \text { if } k_{r}-\left[\sqrt{h_{r}}\right]+1 \leq k \leq k_{r}, \quad r \in \mathbf{N} \\
1, & \text { otherwise. } \\
\leq 1 . &
\end{array}\right.
$$

Theorem 3.7. Let $(X, \mathcal{F}, *)$ be a random 2-normed space. If $x=\left(x_{k}\right)$ be a sequence in $X$, then $S_{\theta\left(\Delta^{n}\right)}^{R 2 N}-\lim x_{k}=\ell$ if and only if there exists a subset $K \subseteq \mathbf{N}$ such that $\delta_{\theta\left(\Delta^{n}\right)}(K)=1$ and $\mathcal{F}_{\Delta^{n}}-\lim x_{k}=\ell$.

Proof. Suppose first that $S_{\theta\left(\Delta^{n}\right)}^{R 2 N}-\lim x_{k}=\ell$. Then for any $t>0, a=$ $1,2,3, \ldots$ and non zero $z \in X$, let

$$
A(a, t)=\left\{k \in \mathbf{N}: \mathcal{F}\left(\Delta^{n} x_{k}-\ell, z ; t\right)>1-\frac{1}{a}\right\}
$$

and

$$
K(a, t)=\left\{k \in \mathbf{N}: \mathcal{F}\left(\Delta^{n} x_{k}-\ell, z ; t\right) \leq 1-\frac{1}{a}\right\}
$$


Since $S_{\theta\left(\Delta^{n}\right)}^{R 2 N}-\lim x_{k}=\ell$ it follows that

$$
\delta_{\theta\left(\Delta^{n}\right)}(K(a, t))=0 .
$$

Now for $t>0$ and $a=1,2,3, \ldots$, we observe that

$$
A(a, t) \supset A(a+1, t)
$$

and

$$
\delta_{\theta\left(\Delta^{n}\right)}(A(a, t))=1 .
$$

Now we have to show that, for $k \in A(a, t), \mathcal{F}_{\Delta^{n}}-\lim x_{k}=\ell$. Suppose that for $k \in A(a, t),\left(x_{k}\right)$ not convergent to $\ell$ with respect to $\mathcal{F}$. Then there exists some $s>0$ such that

$$
\left\{k \in \mathbf{N}: \mathcal{F}\left(\Delta^{n} x_{k}-\ell, z ; t\right) \leq 1-s\right\}
$$

for infinitely many terms $x_{k}$. Let

$$
A(s, t)=\left\{k \in \mathbf{N}: \mathcal{F}\left(\Delta^{n} x_{k}-\ell, z ; t\right)>1-s\right\}
$$

and

$$
s>\frac{1}{a}, a=1,2,3, \ldots
$$

Then we have

$$
\delta_{\theta\left(\Delta^{n}\right)}(A(s, t))=0 .
$$

Furthermore, $A(a, t) \subset A(s, t)$ implies that $\delta_{\theta\left(\Delta^{n}\right)}(A(a, t))=0$, which contradicts $(3.2)$ as $\delta_{\theta\left(\Delta^{n}\right)}(A(a, t))=1$. Hence $\mathcal{F}_{\Delta^{n}}-\lim x_{k}=\ell$.

Conversely, suppose that there exists a subset $K \subseteq \mathbf{N}$ such that $\delta_{\theta\left(\Delta^{n}\right)}(K)=$ 1 and $\mathcal{F}_{\Delta^{n}}-\lim x_{k}=\ell$.

Then for every $\varepsilon>0, t>0$ and non zero $z \in X$, we can find out a positive integer $m=m(\varepsilon, z)$ such that

$$
\mathcal{F}\left(\Delta^{n} x_{k}-\ell, z ; t\right)>1-\varepsilon
$$

for all $k \geq m$. If we take

$$
K(\varepsilon, t)=\left\{k \in \mathbf{N}: \mathcal{F}\left(\Delta^{n} x_{k}-\ell, z ; t\right) \leq 1-\varepsilon\right\}
$$


then it is easy to see that

$$
K(\varepsilon, t) \subset \mathbf{N}-\left\{k_{m+1}, k_{m+2}, \ldots\right\}
$$

and consequently

$$
\delta_{\theta\left(\Delta^{n}\right)}(K(\varepsilon, t)) \leq 1-1 .
$$

Hence $S_{\theta\left(\Delta^{n}\right)}^{R 2 N}-\lim x_{k}=\ell$.

Finally, we establish the Cauchy convergence criteria in random 2normed spaces.

Theorem 3.8. Let $(X, \mathcal{F}, *)$ be a random 2-normed space. Then a sequence $\left(x_{k}\right)$ in $X$ is lacunary $\Delta^{n}$-statistically convergent if and only if it is lacunary $\Delta^{n}$-statistically Cauchy.

Proof. Let $\left(x_{k}\right)$ be a lacunary $\Delta^{n}$-statistically convergent sequence in X. We assume that $S_{\theta\left(\Delta^{n}\right)}^{R 2 N}-\lim x_{k}=\ell$. Let $\varepsilon>0$ be given. Choose $a>0$ such that (3.1) is satisfied. For $t>0$ and for non zero $z \in X$ define

$$
A(a, t)=\left\{k \in \mathbf{N}: \mathcal{F}\left(\Delta^{n} x_{k}-\ell, z ; \frac{t}{2}\right) \leq 1-a\right\}
$$

and

$$
A^{c}(a, t)=\left\{k \in \mathbf{N}: \mathcal{F}\left(\Delta^{n} x_{k}-\ell, z ; \frac{t}{2}\right)>1-a\right\} .
$$

Since $S_{\theta\left(\Delta^{n}\right)}^{R 2 N}-\lim x_{k}=\ell$ it follows that $\delta_{\theta\left(\Delta^{n}\right)}(A(a, t))=0$ and consequently $\delta_{\theta\left(\Delta^{n}\right)}\left(A^{c}(a, t)\right)=1$. Let $p \in A^{c}(a, t)$. Then

$$
\mathcal{F}\left(\Delta^{n} x_{p}-\ell, z ; \frac{t}{2}\right)>1-a .
$$

If we take

$$
B(\varepsilon, t)=\left\{k \in \mathbf{N}: \mathcal{F}\left(\Delta^{n} x_{k}-\Delta^{n} x_{p}, z ; t\right) \leq 1-\varepsilon\right\}
$$

then to prove the result it is sufficient to prove that $B(\varepsilon, t) \subseteq A(a, t)$. Let $k \in B(\varepsilon, t) \cap A^{c}(a, t)$, then for non zero $z \in X$

(3.4) $\mathcal{F}\left(\Delta^{n} x_{k}-\Delta^{n} x_{p}, z ; t\right) \leq 1-\varepsilon$ and $\mathcal{F}\left(\Delta^{n} x_{k}-\ell, z ; \frac{t}{2}\right)>1-a$. 
Then by (3.1), (3.3) and (3.4) we get

$$
\begin{gathered}
1-\varepsilon \geq \mathcal{F}\left(\Delta^{n} x_{n}-\Delta^{n} x_{p}, z ; t\right) \geq \mathcal{F}\left(\Delta^{n} x_{n}-\ell, z ; \frac{t}{2}\right) * \mathcal{F}\left(\Delta^{n} x_{p}-\ell, z ; \frac{t}{2}\right) \\
>(1-a) *(1-a)>(1-\varepsilon)
\end{gathered}
$$

which is not possible. Thus $B(\varepsilon, t) \subset A(a, t)$. Since $\delta_{\theta\left(\Delta^{n}\right)}(A(a, t))=0$, it follows that $\delta_{\theta\left(\Delta^{n}\right)}(B(\varepsilon, t))=0$. This shows that $\left(x_{k}\right)$ is lacunary $\Delta^{n}$ statistically Cauchy.

Conversely, suppose $\left(x_{k}\right)$ is lacunary $\Delta^{n}$-statistically Cauchy but not lacunary $\Delta^{n}$-statistically convergent. Then there exists positive integer $p$ and for non zero $z \in X$ such that

$$
A(\varepsilon, t)=\left\{k \in \mathbf{N}: \mathcal{F}\left(\Delta^{n} x_{k}-\Delta^{n} x_{p}, z ; t\right) \leq 1-\varepsilon\right\} .
$$

then

$$
\delta_{\theta\left(\Delta^{n}\right)}(A(\varepsilon, t))=0
$$

and consequently

$$
\delta_{\theta\left(\Delta^{n}\right)}\left(A^{c}(\varepsilon, t)\right)=1 .
$$

For $a>0$ such that (3.1) is satisfied and we take

$$
B(a, t)=\left\{k \in \mathbf{N}: \mathcal{F}\left(\Delta^{n} x_{k}-\ell, z ; \frac{t}{2}\right)>1-a\right\} .
$$

If $p \in B(a, t)$ then

$$
\mathcal{F}\left(\Delta^{n} x_{p}-\ell, z ; \frac{t}{2}\right)>1-a
$$

Since

$$
\begin{gathered}
\mathcal{F}\left(\Delta^{n} x_{k}-\Delta^{n} x_{p}, z ; t\right) \geq \mathcal{F}\left(\Delta^{n} x_{k}-\ell, z ; \frac{t}{2}\right) * \mathcal{F}\left(\Delta^{n} x_{p}-\ell, z ; \frac{t}{2}\right) \\
>(1-a) *(1-a)>1-\varepsilon
\end{gathered}
$$

then we have

$$
\delta_{\theta\left(\Delta^{n}\right)}\left(\left\{k \in \mathbf{N}: \mathcal{F}\left(\Delta^{n} x_{n}-\Delta^{n} x_{p}, z ; t\right)>1-\varepsilon\right\}\right)=0
$$


i.e. $\delta_{\theta\left(\Delta^{n}\right)}\left(A^{c}(\varepsilon, t)\right)=0$, which contradicts $(3.5)$ as $\delta_{\theta\left(\Delta^{n}\right)}\left(A^{c}(\varepsilon, t)\right)=1$. Hence $\left(x_{k}\right)$ is lacunary $\Delta^{n}$-statistically convergent.

Combining Theorem 3.7 and Theorem 3.8 we get the following corollary.

Corollary 3.9. Let $(X, \mathcal{F}, *)$ be a random 2-normed space and and $x=$ $\left(x_{k}\right)$ be a sequence in $X$. Then the following statements are equivalent:

(a) $x$ is lacunary $\Delta^{n}$-statistically convergent.

(b) $x$ is lacunary $\Delta^{n}$-statistically Cauchy.

(c) there exists a subset $K \subseteq \mathbf{N}$ such that $\delta_{\theta\left(\Delta^{n}\right)}(K)=1$ and $\mathcal{F}_{\Delta^{n}}-\lim x_{k}=$ $\ell$.

Now, we introduce the completeness of random 2-normed spaces.

Definiton 3.9. A random 2-normed space $(X, \mathcal{F}, *)$ is said to be complete if every Cauchy sequence is convergent in $(X, \mathcal{F}, *)$.

As a consequence of the Theorem 3.8, for $n=0$, we define the following definition in random 2-normed spaces.

Definition 3.10. A random 2-normed space $(X, \mathcal{F}, *)$ is said to be $S_{\theta^{-}}$ complete if every $S_{\theta}$-Cauchy sequence is $S_{\theta}$-convergent in $(X, \mathcal{F}, *)$.

Theorem 3.10. Let $\theta$ be a lacunary sequence. Then every random 2normed space $(X, \mathcal{F}, *)$ is $S_{\theta}$-complete but not complete in general.

Proof. First part of the proof of the theorem follows from the Theorem 3.8 , for $n=0$.

To see that random 2-normed space $(X, \mathcal{F}, *)$ is not complete in general, we consider the following example:

Example 3.11. Let $X=(0,1] \times(0,1]$ with the 2-norm $\|x, z\|=\mid x_{1} z_{2}-$ $x_{2} z_{1} \mid, x=\left(x_{1}, x_{2}\right), z=\left(z_{1}, z_{2}\right)$ and $a * b=a b$ for all $a, b \in[0,1]$. Let $\mathcal{F}(x, y ; t)=\frac{t}{t+\|x, y\|}$, for all $x, z \in X, z_{2} \neq 0$, and $t>0$. Then $(X, \mathcal{F}, *)$ is a random 2-normed space but not complete, since the sequence $\left(\frac{1}{k}, \frac{1}{m}\right)$ is Cauchy with respect to $\mathcal{F}$ but not convergent with respect to the present $\mathcal{F}$. This completes the proof of the theorem.

\section{Acknowledgements}

The author expresses his heartfelt gratitude to the anonymous reviewer for such excellent comments and suggestions which have enormously enhanced 
the quality and presentation of this paper.

\section{References}

[1] C. Alsina, B. Schweizer, A. Sklar, Continuity properties of probabilistic norms, J. Math. Anal. Appl. 208, pp. 446452, (1997).

[2] H. Çakalli, Lacunary statistical convergence in topological groups, Indian J. Pure Appl. Math. 26 (2), pp. 113-119, (1995), MR 95m:40016.

[3] J.Connor, M.A. Swardson, Measures and ideals of $C^{*}(X)$, Ann. N.Y. Acad.Sci. 704,, pp. 80-91, (1993).

[4] A. Esi, M. K. Özdemir, Generalized $\Delta^{m}$-Statistical convergence in probabilistic normed space, Jour. Comput. Anal. Appl., 13(5), pp. 923-932, (2011).

[5] A.Esi, M. K. Özdemir, On lacunary statistical convergence in random n-normed space, Annals of Fuzzy Mathematics and Informatics, (To appear)

[6] M.Et and F.Nuray, $\Delta^{m}$-Statistical convergence, Indian J.Pure Appl.Math., 32(6), pp. 961-969, (2001).

[7] H. Fast, Sur la convergence statistique, Colloq. Math. 2, pp. 241-244, (1951).

[8] J. A. Fridy, On statistical convergence, Analysis, 5, pp. 301-313, (1985).

[9] J. A. Fridy, C. Orhan, Lacunary statistical convergence, Pacific J. Math., 160, No. 1, pp. 43-51, (1993), MR 94j:40014.

[10] J. A. Fridy, C. Orhan, Lacunary statistical summability, J. Math. Anal. Appl., 173, pp. 497-504, (1993), MR 95f :40004.

[11] S. Gähler, 2-metrische Raume and ihre topologische Struktur, Math. Nachr. 26, pp. 115-148, (1963).

[12] S. Gähler, Linear 2-normietre Raume, Math. Nachr. 28, pp. 1-43, (1965). 
[13] I . Goleţ, On probabilistic 2-normed spaces, Novi Sad J. Math. 35, pp. 95102, (2006).

[14] M.Gürdal and S. Pehlivan, Statistical convergence in 2-normed spaces, South. Asian Bull. Math.33, pp. 257-264, (2009).

[15] M. Gürdal, S. Pehlivan, The statistical convergence in 2-Banach spaces, Thai J. Math. 2 (1), pp. 107113, (2004).

[16] B. Hazarika, E. Savas, Lacunary statistical convergence in random 2normed space, (communicatted).

[17] S. Karakus, Statistical convergence on probabilistic normed spaces, Math. Commun. 12, pp. 1123, (2007).

[18] S. Karakus, K. Demirci and O. Duman, Statistical convergence on intuitionistic fuzzy normed spaces, Chaos, Solitons and Fractals, 35, pp. 763-769, (2008).

[19] J. Li, Lacunary statistical convergence and inclusion properties between lacunary methods, Internat. J. Math. Math. Sci. 23(3), pp. 175180, (2000), S0161171200001964.

[20] K. Menger, Statistical metrics, Proc. Natl. Acad. Sci. USA 28, pp. 535537, (1942).

[21] H. I. Miller, A measure theoretical subsequence characterization of statistical convergence, Trans. Amer. Math. Soc., 347(5), pp. 18111819, (1995).

[22] M. Mursaleen, Statistical convergence in random 2-normed spaces, Acta Sci. Math.(Szeged), 76(1-2), pp. 101-109, (2010).

[23] M. Mursaleen and S.A. Mohiuddine, On lacunary statistical convergence with respect to the intuitionistic fuzzy normed space, Jour. Comput. Appl. Math., 233(2), pp. 142-149, (2009).

[24] S.A.Mohiuddine, M. Aiyub, Lacunary statistical convergence in random 2-normed space, Appl.Math.Inf.Sci., 6(3), pp. 581-585, (2012).

[25] M. R. S. Rahmat, Lacunary Statistical Convergence on Probabilistic Normed Spaces, Int. J. Open Problems Compt. Math., 2(2), pp. 285292, (2009). 
[26] T. Şalát, On statistical convergence of real numbers, Math. Slovaca, 30, pp. 139-150, (1980).

[27] I. J. Schoenberg, The integrability of certain functions and related summability methods, Amer. Math. Monthly 66, pp. 361-375, (1959).

[28] B. Schweizer, A. Sklar, Statistical metric spaces, Pacific J. Math. 10, pp. 313334, (1960).

[29] B. Schweizer, A. Sklar, Probabilistic Metric Spaces, North Holland, New York- Amsterdam-Oxford, (1983).

[30] C. Sempi, A short and partial history of probabilistic normed spaces, Mediterr. J. Math. 3, pp. 283-300, (2006).

[31] A. N. Serstnev, On the notion of a random normed space, Dokl. Akad. Nauk SSSR 149(, pp. 280-283, (1963).

\section{Bipan Hazarika}

Department of Mathematics,

Rajiv Gandhi University,

Rono Hills,

Doimukh-791112, Arunachal Pradesh,

India

e-mail : bh_rgu@yahoo.co.in 